\title{
Aspects Related Self-Control Function Of Computer Aided Management Control Systems
}

\author{
J.L. Csapo \\ Mechanical Engineering Department, C. Daicoviciu, \\ Technical University of Cluj-Napoca, \\ Cluj-Napoca, Romania
}

\begin{abstract}
Companies operating in geographically dispersed locations require a management control system that allows global access to information, related the performance of different units, allowing all level of management to follow up and intervene when is necessary. Expansion of business to isolated areas with low skilled labor and poor living conditions, which may have less incentive to expatriate employees, the need to remotely manage businesses became more emphasized. Computer Aided Management Control Systems (CAMCS) can offer a viable alternative to physical relocation of high trained employees trough remote assistance from knowledge centers. Ones implemented and validated CAMCS can support the local team and offer up to date information for management. Since physical contact between different management levels is significantly reduced the detail level of information offered by the system need to compensate this gap. Remote management is based on exceptions and the CAMCS need to filter the information in a way to not overload the management and avoid micromanagement, without exposing the company to risks. In order to fulfill this need the systems need to be fortified with self-trigger alert and auto-control mechanism. This paper presents the operative principle of this feature and introduces concepts like Self-Control, Impact Function/Matrix/Tree for CAMCS.
\end{abstract}

Keywords-computer aided management control system; remote management control; self-control; impact function; performance indicators

\section{INTRODUCTION}

Companies operating in capital intensive industries, having activities in geographically dispersed locations require the implementation of integrated and extensive monitoring and management control systems to track and manage assets effectively.

Specific management control applications, which cover only a specific area of the business, do not allow the overall analysis and correlation between different parameters and aspects of the business, like: time utilization of fixed assets, inventory levels, quality performance, economic and financial performance. In the absence of an integrated system which enables automated data processing and analysis correlating these parameters ad hoc, by hand analysis still remains an option, if the necessary infrastructure for data collection is available, but the time needed and costs involved will affect the competitiveness of the unit or organization $[1,4]$.

Rapid data processing and analysis is becoming increasingly important, given that most companies operate in a

\author{
C. Brisan \\ Mechanical Engineering Department, C. Daicoviciu, \\ Technical University of Cluj-Napoca, \\ Cluj-Napoca, Romania
}

market characterized by global competition and high volatility. Delivery time constraint and Just in time delivery arrangements, with significant costs for non-complies, require quick identification of deviations and immediate implementation of corrective actions. Operative management control system which includes all assets and process parameters which can affect operational performance of the company, allows continuous monitoring of efficiency by providing real-time information and enable predictive analysis for realistic planning, obviating stranded deviations.

The paper is structured around four main sections. The part dedicated to Remote Management Control is summarizing the advantages of remoting the management control process and presents the operating principle of the alarm function of the CAMCS. The next two sections are dedicated to Performance Indicators: definition, selection and setting of reference values, summarizing the principles emphasized by the authors as relevant guidelines relate to these processes. The last part of the paper introduces the Self-Control concept, a feature of CAMCS which can significantly improve the reliability of information provided by the control systems.

\section{REMOTE MANAGEMENT CONTROL}

A system that allows global access to information through web-based applications, materialize a tool that facilitates remote management. With the expansion of business in remote areas with low skilled labor and poor living conditions, which may have less incentive to expatriate employees, the possibility of partial remoting of management process is a major advantage of CAMCSs. This type of remote management is based on the concept of management by exceptions where extraordinary circumstances are treated by senior managers and experts from the knowledge centers, based on information available in the control system in collaboration and support of the local management. Exceptions are identified by the deviation from established reference values that are identified automatically by the system and alarm signal is sent to predefined groups. The local team is responsible for managing the unit. Intervention from the center will only occur as a result of specific request or significant deviations from predefined performance indicators, avoiding micro-management without exposing the company to the risk or losing the control of remote units.

Performance indicators having a critical role in the correct functioning of the alarm system and whole CAMCS the correct definition and periodical review, based on clear rules and 
transparent methodology, is an essential role of area responsible [4].

These regular updates of the reference values can be initiated even by the control system itself, based on rules, methodology established (such as best 20\% results of past period; the average of the previous period results, etc.). Budgets validation still remains a task of central management control structure, with the collaboration of the area responsible to avoid any distortion of standards caused by the incidents of the period. Statistical methods, detailed analysis of unexplained variation can be and are recommended to be used in order to eliminate unrepresentative values and to maintain the reference values in a realistic margin.

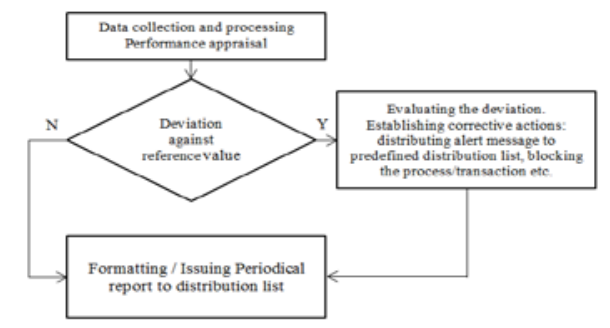

FIGURE I. CAMCS OPERATING FLOWCHART.

Practical experience confirms the effectiveness of management by exceptions and budgeting methods based on the best previous results, leading to a continuous improvement of the targeted process.

\section{PERFORMANCE INDICATORS SELECTION AND DEFINITION OF PERFORMANCE TARGETS}

Performance indicators (PI), as key elements of control process, play an increasingly important role in present economic environment. Enhancing the level of divorce between proprietor and manager function and diversification of capital markets has created the need for homogeneous information package issued periodically to reflect the company's performance, keeping the shareholders up-to-date about company's performance. The sensibility of financial markets to the alarm signals, generated by variations among performance indicators, highlights the importance of correct definition of reference values. The quick changes of business environment require periodical review of the PI set and redefinition of their reference values, based on transparent rules and methodologies.

In case of CAMCS, dedicated for remote management control, when the personal contact between different levels of management is limited, PIs gain a critical role in the proper functioning of the alarm system and the whole structure. With the help of these PI and reference values will be possible to determine whether the different components of the organization works properly, ensuring that progress towards the goals are in time and according to the planned strategy [3].

As objectives and PI must be aligned and interconnected, operational performance and operations level PIs are reflected in higher levels performance. The interconnectedness of PIs need to be traced right from the selection stage and links established between them, in order to create an integrated system, capable of providing continuous performance monitoring at all levels, with the degree of detail required. Having defined the network of connections and impact function (IF) for each indicator CAMCS earn a very important and useful feature: the self-control function, becoming able not only to launch the alarm message if deviation from the defined standard is detected but to identify anomalies related misaligned PIs also. PIs managed through an integrated CAMCS, having defined impact branches (Impact Tree) and proper impact functions become more reliable and can represent a solid base of transparent decision-making process.

A PI to fulfill its role, and become a performance measuring tool, is necessary to define reference values with which they can be compared to determine the degree of deviation and initiate corrective actions depending on the result.

Since this reference values have a crucial role in the correct functioning of the whole system the methodology used for establishing them need to be carefully chosen according to the circumstances.

The methods of establishing performance targets are based on two main principles: analytical computations, bases on technical specification of the process or machine and benchmarking with similar processes/machines or past results of same process/ machine.

Benchmarking on historical results method can be applied for appropriate, well stabilized processes where the reference value for PI can be calculated automatically. The system is programed to calculate these values based on validated formulas (E.g. the best $20 \%$ of the results from the previous period; the best $20 \%$ of results from the previous period or the current standard if the best $20 \%$ average is lower than the current standard, etc.).

To align the performance standards of similar processes from different units of the company, ensuring comparability of results from the whole system, a wider spectrum of comparison can be chosen for benchmarking. Adopting a global reference value, calculated based on the results of the best performing process or machine of the company will assure a global competitiveness and will facilitate continuous improvement and alignment between similar units. (E.g.: Utilization degree of machinery or the number of rejects are recommended to be determined based on the results of the most preformat entity, methodology which will stimulate adoption of best practices in order to achieve the targets).

An additional derivative method, combining numerical methods and historical results, is used in establishing reference values for processes where the effects expected from technological or environmental changes needs to be considered. (E.g.: determining standards after upgrading a machine).

Circumstances may arise when customers or authorities impose performance standards, in which case reference values based on external requirements have to be adopted. (E.g. the number of accidents per 1,000 hours, CO2 emission, etc.)

Standards can be simply imposed by a higher hierarchical level as a contribution needed to higher level goal, without a specific calculation, methodology or justification behind proposed reference values. This instance is not recommended 
since it can compromise the commitment of personnel and can generate dissatisfaction and internal tension between hierarchical levels.

The hierarchical level that sets or validate reference values should be superior to the level which PI is intended to, eliminating conflicts of interest.

\section{The SElF-CONTROL FUnCTION OF CAMCS}

CAMCSs are based on a set of performance indicators by which the system will allow a clear performance diagnosis and enables self-trigger alert and auto-control mechanism, when a significant deviation or inconsistencies between the track parameters are detected.

To define the set of key PI followed by the CAMCS the potential impact on the global PIs have to be identified for each of them. The global PIs are set of basic indicators which reflect the organization's performance, having standard definitions and being internationally recognized (Net Income, Commercial Profit, ROI, etc.). PI list is vast and dynamic, new parameters are introduced whenever the need is identified by the lack of information about a certain deviation generated on an existing PI or technological developments allow the pursuit of new parameters.

PI having different nature their follow up and control is accomplished by determining the deviation in percentage versus the default reference value. Since CAMSC are dedicated mainly for organizations with large structure, where the number of entities is significant and individual PI tracking becomes impossible for higher management levels, a hierarchy of indicators is essential for efficient process control.

To can easily track and represent the hierarchy of different PI is proposed the definition of the Impact Matrix and sketching of Impact tree which represents the significance of each PI based on its potential impact (direct or indirect) on higher level PI.

TABLE I .IMPACT MATRIX.

\begin{tabular}{|c|c|c|c|c|c|c|}
\hline Impact.Matrix & $\mathrm{PI}_{G 1}$ & $\mathrm{PI}_{6:}$ & $\ldots$ & $\mathrm{PI}_{G_{\mathrm{a}}}$ & $\ldots$ & $\mathrm{PI}_{7 \mathrm{~min}}$ \\
\hline $\mathrm{PI}_{\mathrm{CI}}$ & $\cdot$ & 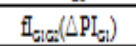 & $\ldots$ & $\mathrm{f}_{\mathrm{gia}}\left(\Delta \mathrm{PI}_{\mathrm{Gi}}\right)$ & $\ldots$ & $\cdot$ \\
\hline $\mathrm{PI}_{\mathrm{CI}}$ & 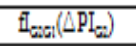 & $\cdot$ & $\ldots$ & & $\ldots$ & $\cdot$ \\
\hline$\ldots$ & $\ldots$ & $\ldots$ & . & $\ldots$ & $\ldots$ & $\ldots$ \\
\hline $\mathrm{PI}_{6}$ & $\mathrm{fl}_{\mathrm{aG}:}\left(\Delta \mathrm{PI}_{\mathrm{E}}\right)$ & $\mathrm{fl}_{a n}\left(\Delta \mathrm{PI}_{a}\right)$ & $\ldots$ & - & $\cdot$ & $\cdot$ \\
\hline$\ldots$ & $\ldots$ & $\ldots$ & $\ldots$ & $\ldots$ & $\cdot$ & $\cdot$ \\
\hline $\mathrm{PI}_{p_{2 n}}$ & 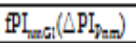 & $\mathrm{API}_{n=c_{1}}\left(\Delta \mathrm{PI}_{\mathrm{h}+n}\right)$ & $\ldots$ & $\mathrm{API}_{\mathrm{na}}\left(\Delta \mathrm{PI}_{\mathrm{h}+\mathrm{n}}\right)$ & $\ldots$ & $\cdot$ \\
\hline
\end{tabular}

fIab(PIa,PIb) - Impact function, defines the impact (deviation) on PIb, caused by the variation in PIa

$$
\begin{aligned}
& \text { PIGx - Global Performance Indicator } \\
& \text { PIPx - Process Performance Indicator }
\end{aligned}
$$

PI are followed individually by the control system, their value being measured or calculated, depending on their nature. The degree of deviation from the reference value is computed directly by comparison to reference value. Computing the expected deviation by numerical methods, based on deviations in related PI and correlated impact functions, and comparing with the values obtained by direct calculation result in a self-lever system that can detect inconsistency between different PI or missing parameters.

The level of a PI is defined by the unit to which it refers and can increase from machine or sub-process level till global level, according to the organizational structure of the company.

A deviation from PI can generate horizontal effects (Intra or Cross-Process impact at the same level) or vertical (Cascade Impact), but only on indicators of higher level.

fIP11P12( $\triangle \mathrm{PIP} 11)$ - Intra process impact: Impact on the different parameters of the same process (E.g. PIp1a -processing temperature. Overheating will affect PIp1b: Energy Consumption)

fIP11Pnm( $\triangle$ PIP11) - Cross process impact: Impact on parallel or additional processes (E.g. PIp1a -processing temperature. Overheating will affect product quality and as a result the subsequent process performance the PIp2a -production per unit of time, since it needs to fix the problem)

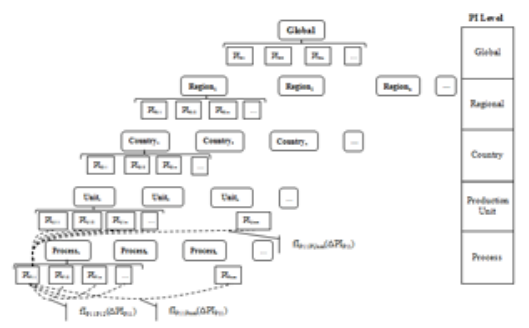

FIGURE II. IMPACT TREE.

fIP11PUnm ( $\triangle$ PIP11) - Cascade Impact: Is the impact of a deviation in PI to higher level PI. The parameters followed by the control system must meet the criteria of relevancy, therefore any deviation on them affects higher level PI (E.g. PIp1a -processing temperature. Overheating will affect consumption of energy therefore will affect PIU1a -the processing cost of the Unit).

\section{CONCLUSIONS}

The technological developments, automation and fast progress of computers and information technology opened a new perspective in management control. The possibility of permanent monitoring of enlarged number of process indicators assures total control and full traceability, revolutionizing the management control and creating new challenges for the human factor that have to deal now with vast amount of information. In order to can prioritize the information, Performance Indicators are defined and selected according to their potential impact to the business. As the number or parameters and Performance Indicators increased correlation between them became necessary in order to avoid anomalies and improve the control system accuracy by auto- control function. CAMCS allowing global access to information through web-based applications, materialize a tool that facilitates remote management of isolated units. With proper Self-Control utility, based on correctly defined Impact functions, and optimally set alarm utility this systems can became a real help in the integration of isolated unit to the corporate management system, contributing 
to efficiency increase and development of local employees without physical presence of the support team.

\section{ACKNOWLEDGMENT}

This paper is supported by the Sectorial Operational Programme Human Resources Development (SOP HRD), ID/134378 financed from the European Social Fund and by the Romanian Government.

\section{REFERENCES}

[1] D. Alberts, R. Hayes, Command and Control in the Information Age, Information on http://www.dodccrp.org/, (2003)

[2] J. Carenys, Management Control Systems: A Historical Perspective, International Journal of Economy, Management and Social Sciences, vol.1, num.1, December 2012, pp. 1-18.

[3] L. Wilkins, W. Ouchi, Efficient cultures exploring the relation-ship between culture and organizational performance, Administrative Science Quarterly, (1983),num.3, pp.469-481.

[4] R. Anthony, V. Govindarajan, Management control systems, 12th ed., McGraw-Hill/Irwin, New York (2007) 